\title{
ASYMPTOTICITY IN TOPOLOGICAL DYNAMICS $\left({ }^{1}\right)$
}

\author{
BY \\ JOHN D. BAUM
}

This paper treats the notion of asymptoticity in topological transformation groups. By a topological transformation group we shall mean a topological group (the phase group) of homeomorphisms of a topological space (the phase space) onto itself, which contains the identity transformation as group identity and which has the property that any two homeomorphisms applied successively yield the same result as their group product applied once. The notion of asymptoticity has been discussed in the past only in the setting where the phase group was either the integers or the one-parameter group of reals (dynamical systems). In such systems two distinct points are said to be positively asymptotic provided their orbits under the set of positive integers (or reals), i.e., under a so-called positive ray, as homeomorphisms become and remain as close as one chooses. A similar notion is available for negative asymptoticity, i.e., asymptoticity under a negative ray; and if a pair of points is both positively and negatively asymptotic, they are said simply to be asymptotic.

It is the purpose of this paper to extend the notion of asymptoticity to transformation groups with a more general structure. In $\$ 1$ we give preliminary definitions, which in the form here presented are due to Gottschalk and Hedlund $[3]\left({ }^{2}\right)$. In $\$ 2$ we define an extended notion of asymptoticity relative to a replete semigroup, i.e., a semigroup of the phase group which contains a translate of each compact set in the phase group. A generalization of $\alpha$ - and $\omega$-limit sets is available to us from [3, sec. 6.33] and is called the $P$-limit set of a point, where $P$ refers to a replete semigroup. We restrict our attention largely to separable generative phase groups and prove a number of theorems about $P$-asymptoticity. It is shown that the $P$-limit set of a point has a minimality property in that it is the smallest set to which the point can be $P$-asymptotic, and further that the point actually is $P$-asymptotic to its $P$-limit set if and only if it does not belong to its $P$-limit set. The principal result of this section is a theorem about unstable transformation groups which states that under suitable hypotheses the existence of $P$-asymptotic points is guaranteed. This theorem contains as a special case a theorem due to Utz [6].

In $\$ 3$ we consider a symbolic transformation group, which is an extension

Presented to the Society, December 28, 1953; received by the editors August 10, 1953.

(1) Except for minor revisions the material of this paper was contained in the author's dissertation presented for the degree of Doctor of Philosophy in Yale University.

(2) Numbers in brackets refer to the bibliography. 
of the Morse minimal set [4]. We define this extension as a symbolic transformation group over the lattice points of the plane as phase group. The properties and structure of this symbolic transformation group are studied in some detail, since it gives a number of examples of asymptoticity described in $\S 2$. We also prove the analogues of theorems known for the Morse minimal set [3, Chap. 12], in particular that this symbolic transformation group is a minimal set and is the orbit closure of a non-periodic, almost periodic point. It is also shown that this minimal set decomposes naturally into two sets, one a set of pairwise separated orbits, the other a set which is the union of pairs of points asymptotic relative to some half plane of the phase group.

\section{Definitions.}

1.01 Definition. Let $T$ be a topological group [5]. $T$ is said to be generative provided that $T$ is abelian and is generated by some compact neighborhood of the identity element, $e$, of $T$.

1.02 REMARK. The assumption that $T$ is generative guarantees that $T$ have a certain structure, namely that $T$ is isomorphic to $C \times I^{n} \times R^{m}$, where $C$ is a compact abelian group, $I$ is the additive group of integers, $R$ the additive group of real numbers, and $n$ and $m$ are non-negative integers [7, p. 110].

1.03 Definition. Let $T$ be a topological group and let $P \subset T$. The set $P$ is called a semigroup provided $P P \subset P$. The set $P$ is called replete provided it contains some bilateral translate of each compact subset of $T$, i.e., if $K \subset T$ is compact, then there exist $t, s \in T$ such that $t K s \subset P$.

1.04 Definition. Let $T$ be a topological group. A subset $A$ of $T$ is said to be $\{$ left $\}\left(^{3}\right)\{$ right $\}$ syndetic in $T$ provided that $T=\{A K\}\{K A\}$ for some compact subset $K$ of $T$. If $T$ is abelian the notions of right and left syndeticism coincide, and we speak simply of syndetic subsets of $T$.

1.05 Definition. Let $X$ be a topological space [2], $T$ a topological group, and $\pi$ a continuous mapping of $X \times T$ into $X$ with the following properties:

(1) $(x, e) \pi=x$ for all $x \in X$, where $e$ is the identity of $T$,

(2) $((x, t) \pi, s) \pi=(x, t s) \pi$ for all $x \in X$ and all $t, s \in T$. The triple $(X, T, \pi)$ is called a transformation group. In the future we shall write $x t$ in place of $(x, t) \pi$.

1.06 Definition. Let $(X, T, \pi)$ be a transformation group, and let $P(x)=\{t \mid t \in T, x t=x\}$, then $P(x)$ is called the period of $x$. The point $x \in X$ is said to be periodic under $T$ provided $P(x)$ is a left syndetic subset of $T$.

1.07 Definition. Let $(X, T, \pi)$ be a transformation group, and let $X$ be a uniform space [8]. Then $(X, T, \pi)$ is called unstable provided there exists an index $\delta$ of $X$ such that for every pair of distinct points $x, y \in X$ there exists $t \in T$ such that $x t \notin y t \delta$. Such an index, $\delta$, is called an index of instability.

(3) Throughout this paper braces are used to indicate alternative readings of the same definition or theorem. All words in the first sets of braces are to be read together, all in the second sets together, etc. For example, in 1.04 "left" is to be read with " $A K$," "right" with "KA." 
1.08 Definition. Let there be distinguished in a topological group $T$ a class of subsets which are called admissible. Let $(X, T, \pi)$ be a transformation group and let $x \in X$, then the group $T$ is said to be recursive at $x$ and the point $x$ is said to be recursive under $T$, or simply recursive provided that if $U$ is a neighborhood of $x$, then there exists an admissible subset $A$ of $T$ such that $x A \subset U$. If the term admissible set is replaced by the term \{left syndetic set\} \{syndetic invariant subgroup \}, then the term recursive is replaced by the term \{almost periodic \} regularly almost periodic .

1.09 Definition. Let $(X, T, \pi)$ be a transformation group, let $X$ be a uniform space, and let $A \subset T$. The points $x, y \in X$ are said to be separated on $A$ provided there exists an index $\gamma$ of $X$ such that $x t \notin y t \gamma$ for all $t \in A$. If $x$ and $y$ are separated on $T$ we simply say $x$ and $y$ are separated.

2. Asymptoticity.

2.01 Standing Notation. Throughout this section $(X, T, \pi)$ will denote a transformation group for which $X$ is a Hausdorff space. Whenever it is stated that $X$ is a unitorm space it will be implicitly assumed that the (Hausdorff) topology of $X$ is that induced by the uniformity.

2.02 Definition. Let $X$ be a uniform space and let $P$ be a replete semigroup in $T$. The points $x, y \in X$ are said to be $P$-asymptotic provided $x \neq y$ and provided that for each index $\gamma$ of $X$ there exists $q \in P$ such that $x q p$ $\in y q p \gamma$ for all $p \in P$.

2.03 Definition. Let $x \in X$, let $A$ be a closed invariant subset of $X$, and let $P$ be a replete semigroup in $T$. The point $x$ is said to be $P$-asymptotic to $A$ provided $x \notin A$ and provided that for each neighborhood $U$ of $A$, there exists $q \in P$ such that $x q P \subset U$.

2.04 Lemma. Let $A$ be a closed invariant set in $X$ such that $x \notin A$. Let $B \subset A$, and let $x$ be P-asymptotic to $B$; then $x$ is P-asymptotic to $A$.

Proof. Clear.

2.05 Lemma. Let $X$ be a compact uniform space. If $A$ is a closed invariant set in $X$ such that $x \notin A$ and if $x$ and $y$ are $P$-asymptotic, $y \in A$, then $x$ is $P$-asymptotic to $A$.

Proof. Let $U$ be any neighborhood of $A$, then there exists $\gamma$, an index of $X$, such that $A \gamma \subset U$. Now since $A$ is invariant and $y \in A, y t \in A$ for all $t \in T$. Thus $y t \gamma \subset U$ for all $t \in T$. Since $x$ and $y$ are $P$-asymptotic, there exists $q \in P$ such that $x q p \in y q p \gamma \subset U$ for all $p \in P$, whence $x q P \subset U$. This completes the proof.

2.06 Definition. Let $x \in X$ and let $P \subset T$. The $P$-limit set of $x$, denoted by $P_{x}$, is defined to be $\bigcap_{t \in T} \mathrm{Cl}(x t P)$. Each point of $P_{x}$ is a $P$-limit point of $x$.

2.07 Remark. Let $x \in X$ and let $P$ be a replete semigroup in $T$ and let $T$ be abelian, then the following are valid statements: 
(1) If $t \in T, P_{x}=P_{x t}=P_{x} t$.

(2) $P_{x}$ is closed and invariant.

(3) $P_{x}=\bigcap_{p \in P} \mathrm{Cl}(x p P) \subset \mathrm{Cl}(x P)$.

(4) If $X$ is compact, then $P_{x} \neq \varnothing$.

Proof. Cf. [3, sec. 6.34].

2.08 Lemma. Let $X$ be a uniform space and let $x$ be P-asymptotic to $A, a$ closed invariant set in $X$; then $P_{x} \subset A$.

Proof. Suppose $y \in P_{x}$ and $y \notin A$. Let $U$ be an open neighborhood of $A$, $V$ a neighborhood of $y$ such that $V \cap U=\varnothing$. Since $x$ is $P$-asymptotic to $A$, there exists $q \in P$ such that $x q P \subset U$. Now since $y \in P_{x}, y \in \mathrm{Cl}(x q P)$, thus $V \cap x q P \neq \varnothing$; therefore let $p_{0} \in P$ such that $x q p_{0} \in V$. Then $x q p_{0} \in V \cap U=\varnothing$, a contradiction, whence $P_{x} \subset A$. This completes the proof.

2.09 Lemma. Let $T$ be a separable generative topological group. Let $P$ be a replete semigroup in $T$. Then there exist elements $\left\{p_{i}\right\}, i=1,2, \cdots$, of $P$ such that if $P_{i}=p_{i} P$, then

(1) $P_{i} \subset P_{i-1}, i=2,3, \cdots$

(2) If $p \in P$, then there exists a positive integer $n$ such that $p P \supset P_{n}$.

Proof. Let $\left\{q_{i}\right\}$ be a countable dense subset of $P$. Define $p_{1}=q_{1} q_{2}, p_{2}$ $=p_{1} q_{3}, \cdots, p_{n}=p_{n-1} q_{n+1}, \cdots$, and define $P_{i}=p_{i} P, i=1,2, \cdots$. Now since $P$ is a semigroup and since $q_{i+1} \in P$, we have $P_{i}=p_{i} P=p_{i-1} q_{i+1} P \subset p_{i-1} P$ $=P_{i-1}$. This completes the proof of (1).

We remark that since $P_{i}=p_{i-1} q_{i+1} P$ and since $p_{i-1} \in P$ we have $q_{i+1} p_{i-1} P$ $\subset g_{i+1} P$, that is, $P_{i} \subset q_{i+1} P$. Now let $p \in P$, then $p P$ is a replete semigroup. Let $U$ be a compact neighborhood of $e$ in $T$, then there exists $t \in T$ such that $t U \subset p P$. Since $P \supset p P \supset t U$, and since $\left\{q_{i}\right\}$ is dense in $P$, there exists a positive integer $n$ such that $q_{n+1} \in t U \subset p P$. Let $q_{n+1}=p r$, where $r \in P$; and let $s \in q_{n+1} P$, whence $s=q_{n+1} u$, where $u \in P$. Then $s=p r u$, and since $P$ is a semigroup, $r u \in P$. Therefore $q_{n+1} P \subset p P$, whence $p P \supset q_{n+1} P \supset P_{n}$. This completes the proof of (2).

2.10 Lemma. Let $T$ be a separable generative group, let $P$ be a replete semigroup in $T$. Then there exist replete semigroups $P_{i}=p_{i} P$, where $p_{i} \in P, i$ $=1,2, \cdots$, such that (1) $P_{i} \subset P_{i-1}$ and (2) $P_{x}=\bigcap_{i=1}^{\infty} \mathrm{Cl}\left(x P_{i}\right)$.

Proof. We remark first that, by $2.07, P_{x}=\bigcap_{p \in P} \mathrm{Cl}(x p P)$. Let $p_{i} \in P$, $i=1,2, \cdots$, be selected so as to satisfy Lemma 2.09 and let $P_{i}=p_{i} P$. Then since $\left\{p_{i}\right\} \subset P$, we have

$$
P_{x}=\bigcap_{p \in P} \mathrm{Cl}(x p P) \subset \bigcap_{i=1}^{\infty} \mathrm{Cl}\left(x p_{i} P\right)=\bigcap_{i=1}^{\infty} \mathrm{Cl}\left(x P_{i}\right) .
$$

Let $p \in P$, then by 2.09 there exists a positive integer $n$ such that $p P \supset P_{n}$, 
whence $P_{x} \supset \bigcap_{i=1}^{\infty} \mathrm{Cl}\left(x P_{i}\right)$. This completes the proof.

2.11 TheOREM. Let $X$ be compact, let $T$ be a separable generative group, and let $P$ be a replete semigroup in $T$. Then $x$ is $P$-asymptotic to $P_{x}$ if and only if $x \notin P_{x}$.

Proof. If $x$ is $P$-asymptotic to $P_{x}$, then by definition $x \notin P_{x}$.

Conversely if $x \notin P_{x}$, since $X$ is compact, by 2.07, $P_{x} \neq \varnothing$ and further $P_{x}$ is a closed invariant set. Let $U$ be any neighborhood of $P_{x}$. By $2.10, P_{x}$ $=\bigcap_{i=1}^{\infty} \mathrm{Cl}\left(x P_{i}\right)=\bigcap_{i=1}^{\infty} \mathrm{Cl}\left(x p_{i} P\right)$, where $p_{i} P \subset p_{i-1} P$, whence $\mathrm{Cl}\left(x p_{i} P\right)$ $C \mathrm{Cl}\left(x p_{i-1} P\right)$. Since $\mathrm{Cl}\left(x p_{i} P\right), i=1,2, \cdots$, are closed nested sets and since $X$ is compact, there exists a positive integer $n$ such that $x p_{n} P \subset U$, where $p_{n} \in P$. This completes the proof.

2.12 Lemma. Let $X$ be compact and let $T$ be a separable generative group. If $A$ is a closed invariant set in $X$ such that $x \notin A$ and if $P_{x} \subset A$, then $x$ is $P$. asymptotic to $A$.

Proof. Use 2.11 and 2.04.

2.13 Theorem. Let $X$ be compact and let $T$ be a separable generative group. Let $P$ be a replete semigroup in $T$, such that $x \notin P_{x}$, and let $\mathfrak{A}=\{A \mid A \subset X$, $A$ closed invariant, $x \notin A, x$ is $P$-asymptotic to $A\}$. Then the following are valid statements:

(1) $P_{x} \in \mathfrak{U}$.

(2) $A \in \mathfrak{A}$ if and only if $P_{x} \subset A$, and $x \notin A$.

(3) $\bigcap_{A \in \mathfrak{U}} A=P_{x}$

Proof. (1) follows from 2.11; (2) follows from 2.08 and 2.12 ; (3) follows from (1) and (2).

2.14 Standing Notation. Let $(X, T, \pi)$ be an unstable transformation group and let $\gamma$ be an index of instability, i.e., for each $x, y \in X, x \neq y$, there exists $t \in T$ such that $x t \notin y t \gamma$. Let $\Delta$ be the diagonal of $X \times X$ and define the product transformation group $(X \times X, T, \rho)$ by $[(x, y), t] \rho=(x t, y t)$.

2.15 REMARK. The only orbits of $(X \times X, T, \rho)$ that lie entirely in $\gamma$ are the orbits of $\Delta$. That is to say if $x, y \in X$ and if $(x t, y t) \in \gamma$ for all $t \in T$, then $x=y$.

2.16 Lemma. Let $X$ be compact, let $P$ be a replete semigroup in $T$, and let $N(\Delta)$ be a neighborhood of $\Delta$ such that $\mathrm{Cl}(N(\Delta)) \subset \gamma$. If $(x p, y p) \in N(\Delta)$ for all $p \in P$, then $x$ and $y$ are $P$-asymptotic, provided $x \neq y$.

Proof. We first show that $(x, y)$ is $P$-asymptotic to $\Delta$ in $(X \times X, T, \rho)$. To show this it is sufficient by 2.12 to show that $P_{(x, y)} \subset \Delta$, where $P_{(x, y)}$ is the $P$-limit set of the point $(x, y)$. By hypothesis $(x p, y p) \in N(\Delta)$ for all $p \in P$, thus $(x, y) p P \subset N(\Delta)$ for all $p \in P$. Thus $\mathrm{Cl}((x, y) p P) \subset \mathrm{Cl}(N(\Delta))$ for all $p \in P$. Therefore $P_{(x, y)} \subset \mathrm{Cl}(N(\Delta)) \subset \gamma$. Now $P_{(x, y)}$ is a closed invariant set, 
thus, by $2.15, P_{(x, y)} \subset \Delta$. This completes the proof that $(x, y)$ is $P$-asymptotic to $\Delta$.

Now let $\delta$ be any index of $X$, then there exists $U$, a neighborhood of $\Delta$, such that $U \subset \delta$; and since $(x, y)$ is $P$-asymptotic to $\Delta$ there exists $q \in P$ such that $(x q p, y q p) \in U \subset \delta$ for all $p \in P$. Thus for each index $\delta$ of $X$ there exists $q \in P$ such that $x q p \in y q p \delta$ for all $p \in P$. This completes the proof.

2.17 Definition. Let $E^{n}$ be euclidean $n$-space with the usual product topology, and let $I^{n}$ be the lattice points of $E^{n}$ with the discrete topology. Points (or vectors) of $E^{n}$ will be designated by $t=\left(t_{i}\right)=\left(t_{1}, \cdots, t_{n}\right)$, and the group operation in $E^{n}$ (or $I^{n}$ ) will be the usual vector addition.

Define $|t|=\left(\sum_{i=1}^{n}\left[t_{i}\right]^{2}\right)^{1 / 2}$ and $t \cdot s=\sum_{i=1}^{n} t_{i} s_{i}$. We remark that the angle $\phi$ between two non-null vectors is given by $\cos \phi=s \cdot t /|s||t|$. Thus two nonnull vectors $s$ and $t$ are parallel provided $s \cdot t=|s||t|$ and orthogonal provided $s \cdot t=0$.

The equation of a hyperplane in $E^{n}$ is given by $\sum_{i=1}^{n} a_{i} t_{i}=c$, where $c$ is a constant, and where we may assume $\sum_{i=1}^{n} a_{i}=1$. The vector $a=\left(a_{i}\right)$ is normal to the hyperplane $\sum_{i=1}^{n} a_{i} t_{i}=0$, and conversely each non-null vector $b=\left(b_{i}\right)$ uniquely determines a hyperplane through the origin, whose equation is $\sum_{i=1}^{n} b_{i} t_{i}=0$.

For $b$ non-null define $P(b)=\left\{t \mid t=\left(t_{i}\right) \in I^{n}, b \cdot t \geqq 0\right\}$. We remark that $P(b)$ is a replete semigroup in $T$, and is called the half space determined by the vector $b$. It is also clear that $P^{-1}(b)=P(-b)=\left\{t \mid t=\left(t_{i}\right) \in I^{n}, b \cdot t \leqq 0\right\}$.

2.18 Theorem. Let $X$ be a compact metric space, dense-in-itself, let $T=I^{n}$ with the usual product topology (i.e., discrete), and let $(X, T, \pi)$ be an unstable transformation group with instability index $\gamma$. Then there exist $x, y \in X$ and $a$ half space $P$ such that $x$ and $y$ are $P$-asymptotic.

Proof. Consider the transformation group $(X \times X, T, \rho)$. Let $\left\{z_{i}\right\}$ be a sequence of points of $N(\Delta)-\Delta$ such that $z_{i} \rightarrow z \in \Delta$, where $N(\Delta)$ is an open neighborhood of $\Delta$ such that $\mathrm{Cl}(N(\Delta)) \subset \gamma$.

Define $A_{i}=\left\{t \mid t \in T, z_{i} t \in N(\Delta)\right\}$. If $A_{i}=\varnothing$, then $z_{i} t \in N(\Delta)$ for all $t \in T$. Thus, by $2.15, z_{i} \in \Delta$, and this contradicts the choice of $z_{i}$. Therefore $A_{i} \neq \varnothing$. Define $r_{i}=\inf _{t \in A_{i}}|t|$. Define, for $r$ real, $S(r)=\{t|t \in T| t \mid,<r\}$ and $S^{*}(r)$ $=\{t|t \in T| t \mid, \leqq r\}$, and let $S_{i}=S\left(r_{i}\right), S_{i}^{*}=S^{*}\left(r_{i}\right)$. Now by definition $S_{i} \subset T$ $-A_{i}$, whence $z_{i} S_{i} \subset N(\Delta)$. Also by definition there exists $t \in S_{i}^{*}$ such that $z_{i} t \notin N(\Delta)$. For each $i$, select $t^{(i)} \in S_{i}^{*}$, so that $z_{i} t^{(i)} \in N(\Delta)$.

Now $\lim _{i \rightarrow \infty} r_{i}=+\infty$. For if $r>0$, then $z S(r) \subset \Delta \subset N(\Delta)$ and, by continuity, there exists an integer $M$ such that $i>M$ implies $z_{i} S(r) \subset N(\Delta)$, whence $r_{i} \geqq r$, provided $i>M$.

Define $s^{(i)}=t^{(i)} /\left|t^{(i)}\right|$. We remark that $t^{(i)} \cdot s^{(i)}=\left|t^{(i)}\right|$. By taking appropriate subsequences we may assume $s^{(i)} \rightarrow s$. Now $P(s)$ is a replete semigroup, and $t^{(i)} \cdot s=t^{(i)} \cdot s^{(i)}-t^{(i)} \cdot\left[s^{(i)}-s\right]=\left|t^{(i)}\right|-\left|t^{(i)}\right|\left|s^{(i)}-s\right| \cos \phi_{i}=\left|t^{(i)}\right|\left\{1-\mid s^{(i)}\right.$ $\left.-s \mid \cos \phi_{i}\right\}$ where $\phi_{i}$ is the angle between $t^{(i)}$ and $s^{(i)}-s$. Since $s^{(i)} \rightarrow s$ and 
$\left|t^{(i)}\right|=r_{i} \rightarrow+\infty$, it follows that $t^{(i)} \cdot s>0$ for $i$ sufficiently large. We can therefore assume that $t^{(i)} \in P(s)$ for all $i$.

Since $X \times X$ is compact, we may also assume that $z_{i} t^{(i)} \rightarrow z^{*}=(x, y)$ where $x, y \in X$. Let $P^{*}(s)=\{t \mid t \in T, t \cdot s>0\}$ and let $u \in P^{*}(s)$. We show that $z^{*} u^{-1} P^{-1}(s) \subset \mathrm{Cl}(N(\Delta))$. Let $p \in P^{-1}(s)$; since $z_{i} t^{(i)} u^{-1} p \rightarrow z^{*} u^{-1} p$, and since $z_{i} S_{i} \subset N(\Delta)$, it will be sufficient to show that $t^{(i)} u^{-1} p \in S_{i}$ for $i$ sufficiently large. Now $u \in P^{*}(s)$ and $p \in P^{-1}(s)$, whence $u \cdot s>0$ and $p \cdot s \leqq 0$; thus we have $\left(u^{-1} p\right)$ $\cdot s=(p-u) \cdot s=p \cdot s-u \cdot s<0$, in other words $u^{-1} p \in P^{*-1}(s)$. Let $t=u^{-1} p$; then $t \cdot s<0$ and $\left|t^{(i)} t\right|=\left|r_{i} s^{(i)}+t\right|=r_{i}\left\{1+\left(2 s^{(i)} \cdot t+t^{2} / r_{i}\right) / r_{i}\right\}^{1 / 2}$. Since $s^{(i)} \cdot t$ $\rightarrow s \cdot t<0$ and since $r_{i} \rightarrow+\infty$, it follows that for sufficiently large $i,\left|t^{(i)} t\right|<r_{i}$, whence $t^{(i)} t=t^{(i)} u^{-1} p \in S_{i}$. Thus $z^{*} u^{-1} P^{-1}(s)=\left(x u^{-1}, y u^{-1}\right) P^{-1}(s) \subset \mathrm{Cl}(N(\Delta))$, and it follows from 2.16 that $x u^{-1}$ and $y u^{-1}$ are $P^{-1}(s)$-asymptotic.

Let $\delta$ be any index of $X$, then there exists $q^{-1} \in P^{-1}(s)$ such that $\left(x u^{-1} q^{-1} p^{-1}, y u^{-1} q^{-1} p^{-1}\right) \in \delta$ for all $p^{-1} \in P^{-1}(s)$. Since $u^{-1} \in P^{-1}(s)$, a replete semigroup in $T$, we have $u^{-1} q^{-1} \in P^{-1}(s)$. Let $v^{-1}=u^{-1} q^{-1}$, then $\left(x v^{-1} p^{-1}\right.$, $\left.y v^{-1} p^{-1}\right) \in \delta$ for all $p^{-1} \in P^{-1}(s)$, whence by definition $x$ and $y$ are $P^{-1}(s)$ asymptotic. This completes the proof.

We now drop the standing notation of 2.14 . If we wish to extend the notion of asymptoticity, i.e., both positive and negative asymptoticity simultaneously, we have several paths open to us. In the remainder of this section we define two such extensions and explore the relation between them. For the remainder of this section we shall assume that the group $T$ is not compact.

2.19 Definition. Let $X$ be a uniform space, let $x, y \in X, x \neq y$. Then $x$ and $y$ are said to be compactly asymptotic provided that for each index $\gamma$ of $X$ there exists a compact set $K \subset T$ such that $t \in K$ implies $(x t, y t) \in \gamma$.

2.20 Definition. Let $X$ be a uniform space, $x, y \in X, x \neq y$; then $x$ and $y$ are said to be totally asymptotic provided $x$ and $y$ are $P$-asymptotic for each replete semigroup $P \subset T, P \neq T$.

2.21 TheOREM. Let $T$ be a generative group, let $X$ be a uniform space, and let $x$ and $y$ be totally asymptotic. Then $x$ and $y$ are compactly asymptotic.

Proof. $T=C \times I^{n} \times R^{m}$ where $n \geqq 0, m \geqq 0$, and $k=n+m>0$, since $T$ is not compact. Let $u_{1}, \cdots, u_{k}$ be the unit points of $I^{m} \times R^{n}$, i.e., the $2^{k}$ distinct points having one coordinate equal to +1 or -1 and the rest 0 . Let $s_{i}=e_{C} \times u_{i}$, whence $e_{C}$ is the identity in $C$. Let $P\left(s_{i}\right)$ be defined as in 2.17 and let $P_{i}=C \times P\left(s_{i}\right)$; then $P_{i}$ is a replete semigroup in $T$ for $i=1$, $2, \cdots, 2 k$.

Let $\delta$ be an index of $X$. Then there exists $p_{i} \in P_{i}$ such that $\left(x p_{i} p, y p_{i} p\right)$ $\in \delta$ for all $p \in P_{i}, i=1,2, \cdots, 2 k$. It is easily shown that $\mathrm{U}_{i=1}^{2 k} p_{i} P_{i}$ covers $T$ except for a compact set $K$ and $t \in T-K$ implies $(x t, y t) \in \delta$. This completes the proof.

2.22 Theorem. Let $X$ be a uniform space, let $T$ be abelian, and let $x$ and $y$ be compactly asymptotic. Then $x$ and $y$ are totally asymptotic. 
Proof. Let $\delta$ be any index of $X$, then by hypotheses there exists a compact set $K \subset T$ such that $t \notin K$ implies $(x t, y t) \in \delta$.

Let $P$ be any replete semigroup in $T, P \neq T$. If $e \in p P$ for each $p \in P$, then $P^{-1} \subset P$, and $T=P P^{-1} \subset P P \subset P$, and $P=T$, contrary to supposition. Thus there exists $p \in P$ such that $e \notin p P$. Let $Q=p P$, then clearly $Q$ is a replete semigroup in $T$. Also for each $q \in Q, q^{-1} Q^{-1} \cap Q=\varnothing$. For suppose the contrary, i.e., there is a $q \in Q$ such that $q^{-1} Q^{-1} \cap Q \neq \varnothing$. Let $s \in q^{-1} Q^{-1} \cap Q$, then $s=q^{-1} q_{1}^{-1}$ and $s=q_{0}$, where $q, q_{0}, q_{1} \in Q$. Thus $q_{0}=q^{-1} q_{1}^{-1}$, and since $Q$ is a semigroup we have $q_{1}^{-1}=q q_{0} \in Q Q \subset Q$, hence $e=q_{1} q_{1}^{-1} \in Q Q \subset Q$, and this contradicts the choice of $Q=p P$.

Now $q^{-1} Q^{-1}$ is a replete semigroup in $T$, and $K \cup e=K^{\prime}$ is compact in $T$; thus there exists $t \in T$ such that $t K^{\prime} \subset q^{-1} Q^{-1}$. Since $e \in K^{\prime}, t \in q^{-1} Q^{-1}$, whence $t=q^{-1} r^{-1}$, where $r \in Q$. Now $q^{-1} r^{-1} K \cap Q=t K \cap Q \subset t K^{\prime} \cap Q \subset q^{-1} Q^{-1} \cap Q=\varnothing$, thus $K \cap r q Q=\varnothing$. Also $Q=p P$, thus $r=p r^{\prime}$ and $q=p^{\prime} q$ where $r^{\prime}, q^{\prime} \in P$, and then $K \cap r q Q=K \cap p r^{\prime} p q^{\prime} p P=\varnothing$. Let $u=p r^{\prime} p q^{\prime} p$, then since $P$ is a semigroup $u \in P$. Furthermore for each $p^{\prime} \in P, u p^{\prime} \notin K$, whence $\left(x u p^{\prime}, y u p^{\prime}\right) \in \delta$. Thus by definition $x$ and $y$ are $P$-asymptotic, and since $P$ was arbitrary $x$ and $y$ are totally asymptotic. This completes the proof.

3. A symbolic transformation group. Throughout this section frequent reference is made to the Morse sequence, $\mu$, and to the bisequence space containing $\mu$. The reference for these is either [4] or [3, chap. 12]. We follow the notation of the latter reference. Most of the proofs of this section are computational in nature and hence are for the most part omitted.

3.01 Definition. Let $S$ be a finite collection of elements, containing at least two elements. $S$ is called the symbol class. Let $I$ be the integers, and $L=I \times I$. The topology in $I$ is the discrete topology, so that the topology in $L$, the lattice points of the plane, is also the discrete topology.

3.02 REMARK. $L$ is a group under the composition: $(p, q)+(r, s)$ $=(p+r, q+s)$. We shall use " + " as the group operation in $L$ as well as in $I$, since the context will always make it clear which is meant.

3.03 Notation. If $A \subset L$ and $(p, q) \in L, A+(p, q)$ will denote the set $\{(r+p, s+q) \mid(r, s) \in A\}$ whereas $A \cup(p, q)$ will denote the set $\{(r, s) \mid(r, s)$ $\in A$ or $(r, s)=(p, q)\}$.

3.04 Definition. An $\{N$-domain $\} \quad\{S$-domain $\}\{E$-domain $\}\{W$-domain $\}\left\{q_{1}\right.$-domain $\}\left\{q_{2}\right.$-domain $\}\left\{q_{3}\right.$-domain $\}\left\{q_{4}\right.$-domain $\}$ rectangle $\}$ is $\{(r, s) \mid s \geqq p$, all $r\}\{(r, s) \mid s \leqq p$, all $r\}\{(r, s) \mid r \geqq p$, all $s\}$ $\{(r, s) \mid r \leqq p$, all $s\}\{(r, s) \mid r \geqq p, s \geqq q\}\{(r, s) \mid r \leqq p, s \geqq q\}$ $\{(r, s) \mid r \leqq p, s \leqq q\}\{(r, s) \mid r \geqq p, s \leqq q\}\{(r, s) \mid p \leqq r \leqq t, q \leqq s \leqq u\}$,

where $p, q, r, s, t, u \in I$ and $p \leqq t, q \leqq u$. A square is a rectangle for which $t-p=u-q$. We shall specify such domains by giving their corner points, that is, for example, the rectangle defined above will be designated by $[(p, q)(t, u)]$; the $N$-domain by $[(-\infty, p)(\infty, \infty)]$; and so on. 
3.05 Definition. An $\mathfrak{A}$-array is a function with domain $\mathfrak{A}$ and range $S$, where $\mathfrak{A}$ is any of the objects defined in 3.04 or $L$ itself. These will usually be called $N$-array, $E$-array, $q_{1}$-array, $L$-array, etc. A rectangle array will be called a block; and if the rectangle is a square, it will be called a square block. If $\mathfrak{A}$ is not a rectangle we shall frequently call an $\mathfrak{U}$-array simply an array, provided it is clear from the context what the domain of the array is. If $A$ is an array, a subrectangle of $A$ is the restriction of $A$ to some rectangle contained in the domain of $A$.

3.06 Definition. Let $D$ be any domain, not a rectangle, then $\left\{D^{-x}\right\}$ $\left\{D^{-y}\right\}$, called the $\{x$-reverse of the domain $D\}\{y$-reverse of the domain $D\}$, is the collection $\{(r,-s)\}\{(-r, s)\}$ where $(r, s) \in D$.

Let $A$ be an array with domain $D$, not a rectangle, then $\left\{A^{-x}\right\}\left\{A^{-y}\right\}$, called the $\{x$-reverse of $A\}\{y$-reverse of $A\}$, is the array $B$ whose domain is $\left\{D^{-x}\right\}\left\{D^{-y}\right\}$ and whose values are defined by $\{B(r,-s)=A(r, s)\}\{B(-r, s)$ $=A(r, s)\}$ where $(r, s) \in D$. If $A$ is a block with domain $D=[(p, u)(q, t)]$, then $\left\{A^{-x}\right\}\left\{A^{-y}\right\}$, called the $\{x$-reverse of $A\}\{y$-reverse of $A\}$, is the block $B$ with domain $D$ whose values are defined by $\{B(r, u+t-j)=A(r, j)\}$ $\{B(p+q-i, s)=A(i, s)\}$ where $\{(r, j)\}\{(i, s)\}$ ranges over the rectangle $D$.

3.07 Definition. If $A$ is an array or block and $(p, q) \in L$, then $A^{(p, q)}$, called the $(p, q)$-translate of $A$, is the array or block $B$ with domain $B$ $=(\operatorname{domain} A)+(p, q)$ and $B(p+i, q+j)=A(i, j)$, where $(i, j) \in \operatorname{domain} A$.

3.08 Definition. Let $A$ and $B$ be arrays or blocks, then $A$ is similar to $B$, denoted by $A \sim B$, if $A^{(p, q)}=B$ for some $(p, q) \in L$. $A$ is contained in $B$, denoted by $A \subset B$, provided $A$ is a restriction of $B$. $A$ contains $B$, denoted by $A \supset B$, provided $A$ is an extension of $B . A$ appears in $B$ provided $C \subset B$ with $C \sim A$. An $(m, n)$-block is a block whose domain is $[(r, s)(u, v)]$ with $u-r=m$ and $v-s=n$, and an $n$-square is an $(n, n)$-block.

3.09 Definition. Let $A_{0}, A_{1}, \cdots$ be a sequence of blocks such that $A_{i} \subset A_{i+1}, i \geqq 0$. $A$, the union of $A_{0}, A_{1}, \cdots=\bigcup_{i=0}^{\infty} A_{i}$, is the array or block $A$ such that domain $A=\bigcup_{i=0}^{\infty}$ domain $A_{i}$ and such that $A_{i} \subset A, i \geqq 0$.

3.10 Definition. Let $A$ be a $\{$ block $\}\left\{q_{1}\right.$-array $\}\left\{q_{2}\right.$-array $\}\left\{q_{3}\right.$-array $\}$ $\left\{q_{4}\right.$-array $\}$ whose domain is $\{[(p, q)(r, s)]\}\{[(p, q)(\infty, \infty)]\}\{[(-\infty, q)$ $(r, \infty)]\}\{[(-\infty,-\infty)(r, s)]\}\{[(p,-\infty)(\infty, s)]\}$, then $\dot{A}$ denotes $\left\{A^{(-p,-q)}\right\}\left\{A^{(-p,-q)}\right\}\left\{A^{(-r,-q)}\right\}\left\{A^{(-r,-s)}\right\}\left\{A^{(-p,-s)}\right\}$.

3.11. Definition. Let $A$ be a $(p, q)$-block, $B$ an $(r, q)$-block, and $C$ a $(p, s)$-block Then by

$$
\{\dot{A} B\}\{B \dot{A}\}\left\{\begin{array}{l}
C \\
\dot{A}
\end{array}\right\}\left\{\begin{array}{l}
\dot{A} \\
C
\end{array}\right\}
$$

is meant the $\{(p+r, q)$-block whose left $(p, q)$-block is $A$ and whose right $(r, q)$-block is similar to $B\}\{(p+r, q)$-block whose left $(r, q)$-block is similar to $B$ and whose right $(p, q)$-block is $A\} \quad\{(p, q+s)$-block whose bottom $(p, q)$-block is $\dot{A}$ and whose top $(p, s)$-block is similar to $C\}\{(p, q+s)$-block 
whose top ( $p, q)$-block is $\dot{A}$ and whose bottom ( $p, s)$-block is similar to $C\}$. Thus if $A_{i}^{j}, i, j=-1,0,1$, are all $n$-squares, it is now clear what is meant by:

$$
\begin{aligned}
& \begin{array}{lll}
A_{-1}^{1} & A_{0}^{1} & A_{1}^{1}
\end{array} \\
& A_{-1}^{0} \dot{A}_{0}^{0} \quad A_{1}^{0} \\
& A_{-1}^{-1} A_{0}^{-1} A_{1}^{-1}
\end{aligned}
$$

3.12 Definition. The $L$-array space, $X=S^{L}$, is the collection of all $L$-arrays. Let $S$ and $L$ be provided with the discrete topology, and $S^{L}$ with the point open or compact index topology.

3.13 Lemma. Define a metric $d: X \times X \rightarrow R$, the reals, as follows: let $x, y \in X$, let $r_{0}=1+\sup \{n \mid x(r, s)=y(r, s)$ for all $r, s \in L$ such that $|r| \leqq n$ and $|s| \leqq n\}$, where we set $n=-1$ if $x(0,0) \neq y(0,0)$; then define $d(x, y)=1 /\left(1+r_{0}\right)$. Then $X$ is dense-in-itself, zero-dimensional, and $d$ is compatible with the topology. In fact $X$ is homeomorphic to the Cantor discontinuum.

3.14 Definition. Let $(p, q) \in L, x \in X$; define $\tau: X \times L \rightarrow X$ by $[x(r, s)$, $(p, q)] \tau=x(r+p, s+q)$. The symbolic L-flow generated by $S$ is the transformation group $(X, L, \tau)$.

3.15 REMARK. Let $x \in X$, then $x$ is periodic if and only if there exist integers $p_{i}, q_{i}, i=1,2$, with the determinant $\left|p_{1} q_{2}\right| \neq 0$ such that $x\left(r+p_{i}, s+q_{i}\right)$ $=x(r, s)$ for $i=1,2$ and all $(r, s) \in L$.

3.16 REMARK. The following statements are equivalent:

(1) $x$ is regularly almost periodic.

(2) If $n>0$ there exist integers $p_{i}, q_{i}, i=1,2$, with $\left|p_{1} q_{2}\right| \neq 0$ such that $x\left(r+p_{1} k+p_{2} j, s+q_{1} k+q_{2} j\right)=x(r, s)$ for $|r| \leqq n,|s| \leqq n$, and all $(k, j) \in L$.

3.17 REMARK. The following statements are pairwise equivalent:

(1) $x$ is almost periodic.

(2) If $n>0$, there exists a syndetic subset $E$ of $L$ such that $x(r+e, s+f)$ $=x(r, s)$ for $|r| \leqq n,|s| \leqq n$, and all $(e, f) \in E$.

(3) If $A$ is a subsquare of $x$, then there exists $k>0$ such that $A$ appears in every $k$-square of $x$.

(4) If $n>0$, there exists $k>0$ such that every $n$-subsquare of $x$ appears in every $k$-subsquare of $x$.

3.18 Remark. If $P$ is any of the domains defined in 3.04, not a rectangle, then $P$ is replete in $L$. Let $x, y \in X$, then $x$ and $y$ are $P$-asymptotic (notation $x \uparrow P y$ ) where $P$ is an $\{N\}\{S\}\{E\}\{W\}\left\{q_{1}\right\}\left\{q_{2}\right\}\left\{q_{3}\right\}\left\{q_{4}\right\}$-domain if and only if there exists an integer $N>0$ such that $m, n \geqq N$ implies $\{x(r, n)$ $=y(r, n)$ for all $r \in I\}\{x(r,-n)=y(r,-n)$ for all $r \in I\}\{x(n, s)=y(n, s)$ for all $s \in I\}\{x(-n, s)=y(-n, s)$ for all $s \in I\}\{x(n, m)=y(n, m)\}\{x(-n, m)$ $=y(-n, m)\}\{x(-n,-m)=y(-n,-m)\}\{x(n,-m)=y(n,-m)\}$.

3.19 Definition. Let $S=[0,1]$. The dual of 0 , denoted $0^{\prime}$, is 1 ; and the dual of 1 , denoted $1^{\prime}$, is 0 . Let $A$ be an array with domain $D ; A^{\prime}$, the $d u a l$ of $A$, 
is the array with domain $D$ such that $A^{\prime}(r, s)=[A(r, s)]^{\prime}$ for all $(r, s) \in D$.

3.20 Definition. Define the sequence of blocks, $Q_{0}, Q_{1}, \cdots$, as follows:

(1) $Q_{0}=\dot{0}$.

(2) If $n>0$,

$$
Q_{n+1}=\begin{aligned}
& Q_{n}^{\prime} Q_{n} \\
& \dot{Q}_{n} Q_{n}^{\prime}
\end{aligned}
$$

(3) Define $Q=\cup_{n=0}^{\infty} Q_{n}$.

(4) Define

$$
\bar{Q}=\begin{aligned}
& \dot{Q} \\
& Q^{-x}
\end{aligned}
$$

(5) Define

$$
\lambda=\bar{Q}^{-y} \dot{\bar{Q}} \quad \text { i.e., } \quad \lambda=\begin{array}{cc}
Q^{-y} & \dot{Q} \\
\left(Q^{-x}\right)^{-y} & Q^{-x} .
\end{array}
$$

3.21 Remark. Let $\mu(n)$ be the Morse sequence, define $x: L \rightarrow S$ as follows:

(1) $x(r, 0)=\mu(r)$.

(2) $x(0, s)=\mu(s)$.

(3) $x(r, s)=\mu(r)$ if $\mu(s)=0, r \neq 0, s \neq 0$.

(4) $x(r, s)=\mu^{\prime}(r)$ if $\mu(s)=1, r \neq 0, s \neq 0$.

Then $x=\lambda$.

3.22 REMARK. The following are valid statements:

(1) $\lambda(r, s)=\mu(s)$ if $\mu(r)=0 . \lambda(r, s)=\mu^{\prime}(s)$ if $\mu(r)=1$.

(2) $\lambda(r, r)=0$.

(3) $\lambda(r-1,-r)=0$.

(4) $\lambda(r, s)=\lambda(s, r)$.

3.23 REMARK. If $\lambda(r, 0)=\lambda(0, s)$, then $\lambda(r, s)=0$. If $\lambda(r, 0) \neq \lambda(0, s)$, then $\lambda(r, s)=1$. In other words, if $\mu(r)=\mu(s)$ then $\lambda(r, s)=0$, and if $\mu(r) \neq \mu(s)$ then $\lambda(r, s)=1$.

3.24 REMARK. For $n>0$ define $\theta(n)=\sum_{i=0}^{\infty} a_{i}$ where $n=\sum_{i=0}^{k} a_{i} 2^{i}, a_{i}=0$ or $1(i=0,1, \cdots, k)$ and define $\theta(0)=0$. If $r, s \geqq 0$, then $\lambda(r, s) \equiv \theta(r)$ $+\theta(s)(\bmod 2)$.

3.25 Definition. Let $A$ be an array or block and let domain $A$ $=[(p, q)(r, s)]$. The $x$ - $Q_{2}$-extension of $A$, denoted by $A^{* x}$, is the array or block such that domain $A^{* x}=[(2 p, q)(2 r+1, s)]$ and $A^{* x}(2 r, t)=A(r, t)$, $A^{* x}(2 r+1, t)=A^{\prime}(r, t)$, where $q \leqq t \leqq s$. The $y$-Q $Q_{2}$-extension of $A$, denoted by $A^{* y}$, is the array or block such that domain $A^{* y}=[(p, 2 q)(r, 2 s+1)]$ and $A^{* y}(u, 2 s)=A(u, s), A^{* v}(u, 2 s+1)=A^{\prime}(u, s)$ where $p \leqq u \leqq r$.

3.26 REMARK. The following are valid statements:

(1) $Q_{n}$ is the lower left corner block of $Q_{n+p}(p>0)$.

(2) $Q_{n}^{-x}=Q_{n}^{-y}=Q_{n}$ if $n$ is even. $Q_{n}^{-x}=Q_{n}^{-y}=Q_{n}^{\prime}$ if $n$ is odd. 
(3) $\left(Q_{n}^{* y}\right)^{* y}=\left(Q_{n}^{* y}\right)^{* x}=Q_{n+1}$.

(4) $\left(Q^{* x}\right)^{* y}=\left(Q^{* y}\right)^{* x}=Q$.

(5) $\lambda^{-x}=\lambda^{-y}=\lambda$.

3.27 REMARK. $\lambda$ is not periodic.

3.28 REMARK. $\lambda$ is almost periodic.

3.29 Remark. Define $N=\mathrm{Cl}(\lambda L)$, then the following statements are valid:

(1) $N$ is a minimal orbit closure under $L$.

(2) If $A$ is a subrectangle of $\lambda$, then $A^{\prime}, A^{-x}, A^{-y}, A^{* x}, A^{* y}$ all occur in $\lambda$.

(3) If $\gamma \in N$, then $\gamma^{\prime}, \gamma^{-x}, \gamma^{-y}, \gamma^{* x}, \gamma^{* y} \in N$.

3.30 Remark. Let $\kappa=\left(\lambda^{* x}\right)^{* y}$. The five operations, ', $-x,-y,{ }^{*} x,{ }^{*} y$ yield exactly eight distinct arrays, namely $\lambda, \lambda^{\prime}, \kappa, \kappa^{\prime}, \lambda^{* x}, \lambda^{* y}, \kappa^{\prime * x}, \kappa^{\prime * y}$. The following relations pertain: $\lambda=\lambda^{-x}=\lambda^{-y}, \quad \lambda^{\prime}=\lambda^{\prime-x}=\lambda^{\prime-y}, \quad \lambda^{* x}=\kappa^{* y}$ $=\left(\lambda^{* x}\right)^{-x}, \quad \lambda^{* y}=\kappa^{* x}=\left(\lambda^{* y}\right)^{-y}, \quad \kappa=\left(\lambda^{* x}\right)^{* y}=\left(\lambda^{* y}\right)^{* x}, \quad \kappa^{\prime}=\kappa^{-x}=\kappa^{-y}, \quad \kappa^{* * x}$ $=\left(\kappa^{\prime * x}\right)^{-x}=\left(\lambda^{* y}\right)^{-x}=\lambda^{\prime * y}=\lambda^{* y^{\prime}}, \kappa^{\prime * y}=\left(\kappa^{\prime * y}\right)^{-x}=\left(\lambda^{* x}\right)^{-y}=\lambda^{\prime * x}=\lambda^{* x^{\prime}}$. In summary:

$$
\begin{aligned}
& \lambda=\begin{array}{cl}
Q^{-y} & \dot{Q} \\
\left(Q^{-x}\right)^{-y} & Q^{-x},
\end{array} \quad \lambda^{\prime}=\begin{array}{c}
Q^{-y^{\prime}} \quad \dot{Q}^{\prime} \\
\left(Q^{-x}\right)^{-y^{\prime}} Q^{-x^{\prime}},
\end{array} \quad \lambda^{* x}=\begin{array}{c}
Q^{-y^{\prime}} \quad \dot{Q} \\
\left(Q^{-x}\right)^{-y^{\prime}}
\end{array} Q^{-x}, \\
& \lambda^{* y}=\begin{array}{cl}
Q^{-y} \quad \dot{Q} \\
\left(Q^{-x}\right)^{-y^{\prime}} Q^{-x^{\prime}},
\end{array} \quad \kappa=\begin{array}{cc}
Q^{-y^{\prime}} & \dot{Q} \\
\left(Q^{-x}\right)^{-y} & Q^{-x^{\prime}},
\end{array} \quad \kappa^{\prime}=\begin{array}{cc}
Q^{-y} & \dot{Q}^{\prime} \\
\left(Q^{-x}\right)^{-y^{\prime}} & Q^{-x},
\end{array} \\
& \kappa^{\prime * x}=\begin{array}{cc}
Q^{-y^{\prime}} & \dot{Q}^{\prime} \\
\left(Q^{-x}\right)^{-y} & Q^{-x},
\end{array} \quad \kappa^{\prime * y}=\begin{array}{cc}
Q^{-y} & \dot{Q}^{\prime} \\
\left(Q^{-x}\right)^{-y} & Q^{-x^{\prime}} .
\end{array}
\end{aligned}
$$

3.31 REMARK. The following statements are valid:

(1) The eight arrays of $3.30 \in N$.

(2) $\lambda$ and $\lambda^{\prime}$ are separated. $\kappa$ and $\kappa^{\prime}$ are separated. $\lambda^{* y}$ and $\kappa^{\prime * x}$ are separated. $\lambda^{* x}$ and $\kappa^{\prime * y}$ are separated.

3.32 Remark. Let $E=\{(r, s) \mid r \geqq 0\}, W=\{(r, s) \mid r \leqq 0\}, N=\{(r, s) \mid s$ $\geqq 0\}, S=\{(r, s) \mid s \leqq 0\}, q_{1}=\{(r, s) \mid r \geqq 0, s \geqq 0\}, q_{2}=\{(r, s) \mid r \leqq 0, s \geqq 0\}$, $q_{3}=\{(r, s) \mid r \leqq 0, s \leqq 0\}, q_{4}=\{(r, s) \mid r \geqq 0, s \leqq 0\}$, then

(1) $E, W, N, S, q_{1}, q_{2}, q_{3}, q_{4}$ are all replete semigroups in $L$.

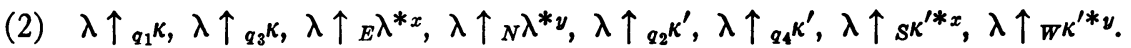

(3) $\lambda^{\prime} \uparrow_{q_{2}} \kappa, \lambda^{\prime} \uparrow_{q_{4}} k, \lambda^{\prime} \uparrow_{w} \lambda^{* x}, \lambda^{\prime} \uparrow_{s} \lambda^{* y}, \lambda^{\prime} \uparrow_{q_{1}} \kappa^{\prime}, \lambda^{\prime} \uparrow_{q_{3}} k^{\prime}, \lambda^{\prime} \uparrow_{N} \kappa^{\prime * x}, \lambda^{\prime} \uparrow_{E} \kappa^{\prime * y}$.

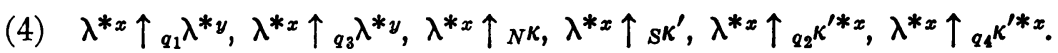

(5) $\lambda^{* y} \uparrow_{E k}, \lambda^{* y} \uparrow_{W K^{\prime}}, \lambda^{* y} \uparrow_{{ }_{2} K^{k}}{ }^{* y}, \lambda^{* y} \uparrow_{q_{1} K^{\prime}}{ }^{* y}$.

(6) $\kappa \uparrow \varpi \kappa^{\prime * x}, \kappa \uparrow s \kappa^{\prime * y}$.

(7) $\kappa^{\prime} \uparrow_{E} \kappa^{\prime * x}, \kappa^{\prime} \uparrow_{N} \kappa^{\prime * y}$.

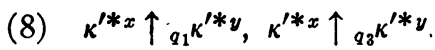


(9) All pairs of arrays in (2)-(8) above are separated on the complement of the set on which they are asymptotic.

3.33 Definition. Let $M$ be the orbit closure of the Morse sequence. Let $Y$ be the bisequence space containing $M$, and let $x, y \in Y$, such that $x(0)=y(0)$. Construct $z$, an $L$-array, as follows:

$$
z(r, s)=\left\{\begin{array}{lll}
x(r) & \text { if } & y(s)=y(0) \\
x^{\prime}(r) & \text { if } & y(s) \neq y(0)
\end{array}\right.
$$

Our notation will be $z=[x, y]$. Let $\mathfrak{N}$ be the collection of all such $z=[x, y]$ as $x$ and $y$ range over $M$.

3.34 REMARK. The following are valid statements:
(1) $\quad \lambda=[\mu, \mu]$
(3) $\lambda^{* x}=[\nu, \mu]$,
(5) $\quad \kappa=[\nu, \nu]$,
(7) $\kappa^{\prime * x}=\left[\mu^{\prime}, \nu^{\prime}\right]$

(2) $\lambda^{\prime}=\left[\mu^{\prime}, \mu^{\prime}\right]$,

(4) $\lambda^{*} y=[\mu, \nu]$

(6) $\kappa^{\prime}=\left[\nu^{\prime}, \nu^{\prime}\right]$,

(8) $\kappa^{\prime * y}=\left[\nu^{\prime}, \mu^{\prime}\right]$,

where $\nu$ is defined as in [3, sec. 12.35].

\subsection{Theorem. $\mathfrak{R}=N$.}

Proof. Let $z$ be an $L$-array and for $k$ integral let $z \mid[(r, s), s=k]=x_{k}$, and $z \mid[(r, s), r=k]=y_{k}$. It is readily verified that $z \in \mathfrak{N}$ if and only if there exists $x, y \in M$ such that for all $k, x_{k}=x$ or $x^{\prime}$ and $y_{k}=y$ or $y^{\prime}$. Since $M$ is closed and invariant under $I$, it follows that $\mathfrak{R}$ is closed and invariant under $L$. Since $\lambda=[\mu, \mu] \in N \cap \mathfrak{R}$, we infer that $N \subset \mathfrak{R}$.

Let $z \in \mathfrak{R}$ and let $m$ be any positive integer. There exists a pair $(r, s)$ of integers such that $\lambda=[\mu, \mu]$ has the property that $\lambda(r+i, s)=z(i, 0)$ and $\lambda(r, s+i)=z(0, i)$ for $|i| \leqq m$. It follows that $\lambda(r+i, s+j)=z(i, j)$ for $|i|$ $\leqq m$ and $|j| \leqq m$. Thus the orbit of $\lambda$ under $L$ is dense in $\mathfrak{R}$ and we infer that $\mathfrak{R \subset}$. This completes the proof.

3.36 Remark. If $z=\left[x_{0}, y_{0}\right]$, then $z^{\prime}=\left[x_{0}^{\prime}, y_{0}^{\prime}\right]$.

3.37 Definition. Let $Y$ be the bisequence space containing $M$, the orbit closure of the Morse sequence. Let $\pi: Y \rightarrow Y$ be defined as follows:

$$
(x \pi)(i)=\left\{\begin{array}{l}
0 \text { if } x(i-1) \neq x(i), \\
1 \text { if } x(i-1)=x(i) \text { for all } x \in Y \text { and all } i \in I .
\end{array}\right.
$$

Define $\eta=\mu \pi, \zeta=\nu \pi, H=M \pi$. Now define the mapping $\rho: N \rightarrow X$ as follows: let $z \in N=\mathfrak{N}, z=\left[x_{0}, y_{0}\right]$, where $x_{0}$ and $y_{0} \in M . z \rho=\left[x_{0} \pi, y_{0} \pi\right]$. Let $N \rho=G$.

3.38 REMARK. The following are valid statements:

(1) $\rho$ is an exactly two-to-one continuous mapping of $N$ onto $G$.

(2) $z \rho=z^{\prime} \rho$ and $z \rho \rho^{-1}=\left\{z, z^{\prime}\right\}$.

(3) $z t \rho=z \rho t$.

(4) If $z$ is recursive then $z \rho$ is recursive. 
(5) If $z \uparrow_{P} w$, then $z \rho \uparrow_{P} w \rho$.

(6) $G$ is a minimal orbit closure under $L$.

3.39 REMARK. Let $\mathfrak{M}=\left\{z_{0} L \mid z_{0} \in N=\mathfrak{N}, z_{0}=\left[x_{0}, y_{0}\right]\right.$ and $x_{0}=\mu, \mu^{\prime}, \nu$, or $\nu^{\prime}$ or $y_{0}=\mu, \mu^{\prime}, \nu$, or $\nu^{\prime}$, or both $\}$. Then

(1) $\mathfrak{M} \subset \mathfrak{N}$.

(2) $(\mathfrak{N}-\mathfrak{M}) \rho=\mathfrak{N} \rho-\mathfrak{M} \rho$.

(3) If $z \rho \in(\mathfrak{N}-\mathfrak{M}) \rho$ then $z \rho$ is regularly almost periodic.

Proof. (3) Let $z=\left[x_{0}, y_{0}\right], x_{0}$ and $y_{0} \neq \mu, \mu^{\prime}, \nu$, or $\nu^{\prime} . z \rho=\left[x_{0} \pi, y_{0} \pi\right]$. Now both $x_{0} \pi$ and $y_{0} \pi$ belong to the complement of the union of the orbits of $\eta$ and $\zeta$ in $H$ and by [3, sec. 12.53] are regularly almost periodic.

Let $n>0$, then there exist integers $p>0$ and $q>0$ such that $x_{0} \pi(i+p j)$ $=x_{0} \pi(i)$ for $|i| \leqq n$ and all $j \in I$ and $y_{0} \pi(i+q j)=y_{0} \pi(i)$ for $|i| \leqq n$ and all $j \in I$. It follows that $z \rho(r+p j+p k, s+q j+2 q k)=z \rho(r, s)$ for all $|r| \leqq n,|s|$ $\leqq n$ and all $(j, k) \in L$. By $3.16, z \rho$ is regularly almost periodic. This completes the proof.

3.40 REMARK. If $z_{1}, z_{2} \in \mathfrak{N}-\mathfrak{M}$, then $z_{1}$ and $z_{2}$ are separated.

Proof. Suppose the contrary, i.e. $z_{1}$ and $z_{2}$ are nonseparated. But $z_{1} \rho$ and $z_{2} \rho$ are in $(\mathfrak{N}-\mathfrak{M}) \rho=\mathfrak{N} \rho-\mathfrak{M} \rho$, and by $3.39(3), z_{1} \rho$ and $z_{2} \rho$ are regularly almost periodic. Thus by $[3$, sec. 10.43$]$ we have a contradiction. Therefore $z_{1}$ and $z_{2}$ are separated. This completes the proof.

3.41 REMARK. Let $N, E, W$, and $S$ be the subsets of $L$ defined in 3.32 and let $z \in \mathfrak{M}$. Then there exists $z_{0} \in \mathfrak{M}$ such that $z \uparrow_{P} z_{0}, P=N, E, W$, or $S$.

Proof. Let $z=[x, y]$. Now either $x$ or $y=\mu, \mu^{\prime}, \nu$, or $\nu^{\prime}$ or possibly both. We suppose that $x=\mu$, the proofs being similar for the other cases. Let $x_{0}=\nu$ and let $y_{0}=y$. Let $z_{0}=\left[x_{0}, y_{0}\right]$. We remark that $z \neq z_{0}$, since $\mu \neq \nu$ and also by the construction of $z_{0}$, and due to the fact that $\mu(r)=\nu(r)$ for $r \geqq 0$, we have that $z(r, s)=z_{0}(r, s)$ for $r \geqq 0$ and all $s \in I$. By 3.18 this implies that $z \uparrow_{E} z_{0}$. This completes the proof.

\section{BiBLIOGRAPHY}

1. P. Alexandroff and H. Hopf, Topologie, Berlin, 1935.

2. N. Bourbaki, Topologie génêrale, Actualités Scientifiques et Industrielles, no. $858=1142$, Paris, 1951.

3. W. H. Gottschalk and G. A. Hedlund, Topological dynamics [forthcoming].

4. M. Morse and G. A. Hedlund, Symbolic dynamics, Amer. J. Math. vol. 60 (1938) pp. 815-864.

5. L. Pontrjagin, Topological groups, Princeton, 1939. 774.

6. W. R. Utz, Unstable homeomorphisms, Proc. Amer. Math. Soc. vol. 1 (1950) pp. 769-

7. A. Weil, L'intégration dans les groupes topologiques et ses applications, Actualités Scientifiques et Industrielles, no. 869, Paris, 1940.

8. - Sur les espaces à structure uniforme et sur la topologie gênérale, Actualités Scientifiques et Industrielles, no. 551, Paris, 1938.

YaLe UNIVERSity,

New Haven, Conn. 